\title{
Phytothérapie et prise en charge des séquelles des accidents vasculaires cérébraux en Côte d'Ivoire
}

\author{
PIBA Serge Cherry ${ }^{1}$, KONE N'gana Losséni ${ }^{2}$, KONAN Kouassi Pierre Alain ${ }^{3}$, SOA Jeanol \\ Duvalier $^{2}$, KOUAME Amoin Gervaise ${ }^{2}$, YAO Kouamé Barthélémy ${ }^{4}$, KROA Ehoulé ${ }^{4}$, TRA \\ Bi Fezan Honora ${ }^{2}$ \\ ${ }^{1}$ Université de Man, UFR Ingénierie Agronomique Forestière et Environnementale, BP 20 Man. Auteur correspondant, Mail: \\ sergepiba@gmail.fr \\ ${ }^{2}$ Université Nangui Abrogoua, UFR Sciences de la Nature, 02 BP 801 Abidjan 02.Mail:nganalosskone@gmail.com. \\ jeanolsoa@gmail.com,gervaka@gmail.com,trabifezan@yahoo.fr \\ ${ }^{3}$ Université Nangui Abrogoua, Laboratoire de Chimie Bioorganique des Substances Naturelles, 02 BP 801 Abidjan 02. \\ Mail:pierredesprogres@gmail.com \\ ${ }^{4}$ Programme National de Promotion de la Médecine Traditionnelle (PNPMT), Tél. : 20332781. Mail: \\ yaok0712@gmail.com,ek.roa2002@yahoo.fr
}

Mots clés : AVC, séquelles, préparations phytothérapeutiques, phytochimie, toxicité.

Keywords: Stroke, sequelae, herbal preparations, phytochemistry, toxicity.

Publication date 30/11/2020, http://m.elewa.org/Journals/about-japs/

\section{RESUME}

Les Accidents Vasculaires Cérébraux (AVC) sont une urgence de santé publique. Le présent travail vise à évaluer, dans la pharmacopée ivoirienne, les connaissances des phytothérapeutes puis à identifier les préparations médicamenteuses utilisées dans le traitement des séquelles des AVC, à déterminer leurs caractéristiques ethnopharmacologiques, phytochimiques, leur potentiel antioxydant et leur toxicité pour une meilleure prise en charge des malades. Les résultats des enquêtes réalisées auprès de 20 phytothérapeutes du Programme National de Promotion de la Médecine Traditionnelle (PNPMT) ont montré que l'hypertension artérielle est la principale cause des AVC et l'hémiplégie, la séquelle majeure. Parmi les 36 préparations collectées, 10 parmi les extraits aqueux ont fait l'objet de tests. Les résultats indiquent la présence de polyphénols, de flavonoïdes, de tanins, de stérols et de saponosides dans toutes les préparations. Daoutra Fort et Daoutra 3 sont celles qui ont présenté les teneurs les plus élevées en polyphénols $(173913,8$ et $151456,9 \mu \mathrm{g}$ EAG/g MS) et en flavonoïdes $(77,13 \%$ et 75,07 $\%)$. Les résultats indiquent également que Ballo $\left(\mathrm{CI}_{50}=0,0280 \mathrm{mg} / \mathrm{mL}\right)$, Daoutra Fort $(0,0409$ $\mathrm{mg} / \mathrm{mL})$ et Daoutra $3(0,0593 \mathrm{mg} / \mathrm{mL})$ présentent d'importants potentiels antioxydants. Les tests de toxicité aigüe ont indiqué que, pour les préparations, la $\mathrm{DL}_{50}$ est supérieure à 5000 $\mathrm{mg} / \mathrm{kg}$ de pc. Elles peuvent être considérées comme non toxiques par voie orale.

\begin{abstract}
Stroke are a public health emergency. The present work aims to evaluate, in the Ivorian pharmacopoeia, the knowledge of phytotherapists then to identify the medicinal preparations used in the treatment of the sequelae of stroke, to determine their ethnopharmacological and phytochemical characteristics, their antioxidant potential and their toxicity for a better taking in charge of the sick. The results of surveys carried out with 20 phytotherapists from the National Program for the Promotion of Traditional Medicine (PNPMT) showed that high blood pressure is the main cause of stroke and hemiplegia, the major sequel. Of the 36
\end{abstract}


preparations collected, 10 of the aqueous extracts were tested. The results indicate the presence of polyphenols, flavonoids, tannins, sterols and saponosides in all preparations. Daoutra Fort and Daoutra 3 are those which presented the highest contents of polyphenols $(173,913.8$ and $151,456.9 \mu \mathrm{g}$ EAG / $\mathrm{g}$ DM) and of flavonoids $(77.13 \%$ and $75.07 \%)$. The results also indicate that Ballo (IC50 $=0.0280 \mathrm{mg} / \mathrm{mL})$, Daoutra Fort $(0.0409 \mathrm{mg} / \mathrm{mL})$ and Daoutra $3(0.0593 \mathrm{mg} / \mathrm{mL})$ exhibit significant antioxidant potential. Acute toxicity testing indicated that for preparations the $\mathrm{DL}_{50}$ is greater than $5000 \mathrm{mg} / \mathrm{kg}$ bw. They can be considered nontoxic by the oral route.

\section{INTRODUCTION}

Les troubles thromboemboliques tels que les embolies pulmonaires, la thrombose veineuse profonde, les crises cardiaques et les Accidents Vasculaires Cérébraux (AVC) étaient les principales causes de morbidité et de mortalité dans les pays développés (Dickneite et al., 1995 ; Go et al., 2014), avant la pandémie liée au coronavirus (Covid-19). Les AVC constituent une cause importante de cette mortalité et la première cause d'handicap moteur dans le monde (Bouleti et al., 2014). En médecine clinique, les récupérations motrices sont incomplètes pour $2 / 3$ des personnes et une assistance humaine est indispensable pour 25 à 30\% d'entre-elles (Hendricks et al., 2002). Dans le cas où une paralysie se développe, moins de $15 \%$ des patients ont une récupération motrice complète. La médecine traditionnelle chinoise, riche d'une expérience clinique vieille de plusieurs millénaires, a montré son efficacité en clinique sur des patients victimes d'accidents vasculaires cérébraux (Quintard, 2014). L'efficacité des préparations a été mise en évidence par des tests de survie et de récupération motrice. Les effets neuroprotecteurs et neurorégénérateurs ont été mis en évidence sur deux modèles expérimentaux de " cérébro-lésion ", l'un ischémique et l'autre traumatique (Quintard, 2014). Ainsi, pour pallier aux limites de la médecine clinique face aux séquelles des AVC, aux nombreux effets secondaires liés l'utilisation d'anticoagulants et autres agents thrombolytiques (Leys et al., 2009 ; Bouleti et al., 2014), l'utilisation des préparations à base de plantes dans le traitement des Maladies cardiovasculaires est, de plus en plus, courante. L'activité thrombolytique, antithrombotique, antioxydante de plusieurs plantes a déjà été évaluée (Kee et al., 2008 ; Daud et al., 2013, LunaVázquez et al., 2013 ; Tanelli et al., 2017). Les propriétés antioxydantes des certains métabolites secondaires sont indispensables dans la régénération des tissus par la réduction du stress oxydant lié à la survenue des AVC (Quintar, 2014). Par exemple, selon Kee et al. (2008), les tanins présentent des propriétés anticoagulantes. Plusieurs auteurs (Proteggente et al., 2011; Kawakami, 2011) ont mis en évidence des propriétés vasodilatatrices et antihypertensives de certains flavonoïdes. Des polyphénols présentent, en plus des propriétés antioxydantes, d'autres propriétés vasodilatatrices, anti-thrombotiques et antiinflammatoires (Manach et al., 2005). En Afrique où $80 \%$ de la population à recours à la médecine traditionnelle pour ses soins de santé, il est important de mettre à leur disposition des plantes ou des préparations phytothérapeutiques pour aider à la prise en charge des malades victimes des AVC. Cette étude vise à évaluer les connaissances des phytothérapeutes, à identifier dans la pharmacopée ivoirienne, les préparations médicamenteuses utilisées dans le traitement des séquelles des AVC, à déterminer leurs caractéristiques ethnopharmacologiques, phytochimiques, leur potentiel antioxydant et leur toxicité pour une meilleure prise en charge des malades. 


\section{METHODOLOGIE}

\subsection{Enquêtes ethnopharmacologiques :} Les personnes ressources de cette étude sont des phytothérapeutes sélectionnés dans le cadre d'une collaboration avec le Programme National de Promotion de la Médecine Traditionnelle (PNPMT), une structure du Ministère de la Santé en Côte d'Ivoire. Elles sont affiliées et membres du PNPMT et ont, au moins, une préparation médicamenteuse utilisée dans le traitement des séquelles des AVC. Un focus groupe a permis d'expliquer les finalités de l'étude et d'encadrer la confidentialité liée aux noms des plantes utilisées dans les préparations. Les interviews ont été réalisées sur 7 mois, de juillet 2018 à janvier 2019, dans la localité de résidence des praticiens, à Abidjan, capitale économique, pour la majorité, et dans d'autres villes de l'intérieur du pays (Dabou, Agboville, Gagnoa, Daloa, Yamoussoukro et Korhogo) pour les autres (Figure 1). Les enquêtes ont été menées en français, sur la base d'un questionnaire, lors d'entretiens semi-structurés. Les rubriques concernées étaient les informations sur l’identité du phytothérapeute, ses connaissances sur les AVC et sur l'utilisation des plantes médicinales pour traiter les séquelles des cérébro-lésions.

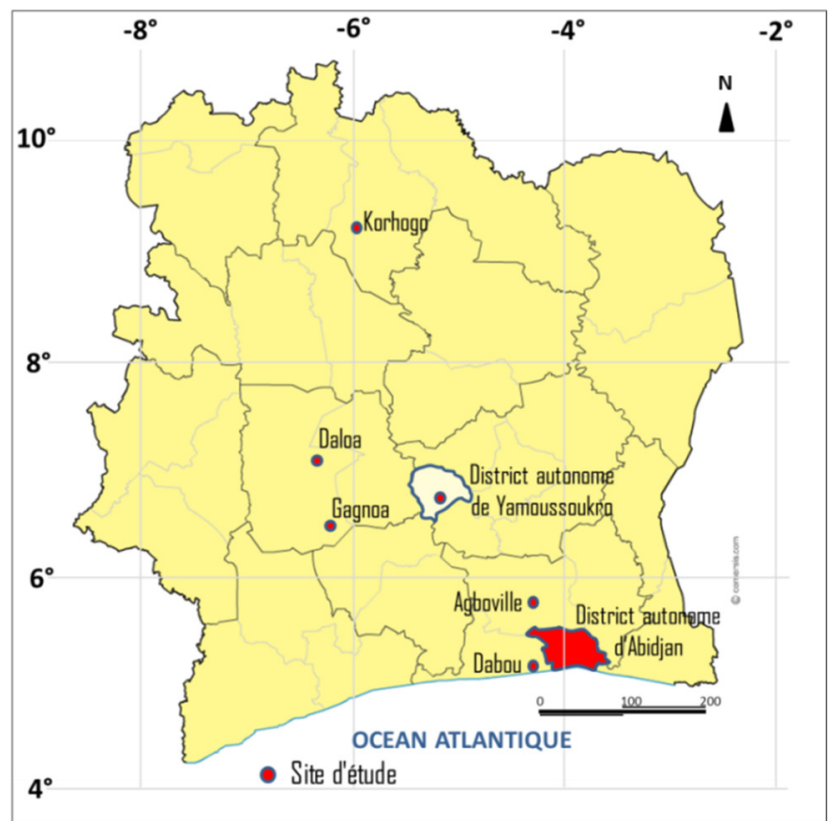

Figure 1: Situation géographique de la Côte d'Ivoire indiquant les localités de résidence des phytothérapeutes retenus pour l'étude

\subsection{Choix des préparations} médicamenteuses à tester et production des extraits bruts: Dix préparations médicamenteuses ont été sélectionnées pour les tests. Leur nature était le premier critère. Celles qui ont été retenues étaient des solutions aqueuses utilisées généralement seules pour le traitement étiologique et administrées oralement. Le second critère était de la réputation des praticiens (parution dans des journaux, distinctions obtenues). Les préparations ont été directement lyophilisées pour produire des extraits bruts qui ont été pesés et stockés dans des récipients étanches puis conservés à température constante $\left(4^{\circ} \mathrm{C}\right)$.

3.3 Criblage phytochimique : Le criblage phytochimique a été réalisé suivant les procédures analytiques décrites par Harbome et al. (1998). Les polyphénols et les tanins ont été mis en évidence par la réaction au chlorure ferrique $\left(\mathrm{FeCl}_{3}\right)$. Les flavonoïdes sont détectés par la réaction au trichlorure d'aluminium, les 
stérols par l'acide sulfurique concentré $\left(\mathrm{H}_{2} \mathrm{SO}_{4}\right)$, les alcaloïdes par le réactif de Dragendor'ff puis les saponosides par le test de la mousse. Ces différents groupes de composés chimiques ont été retenus pour leur importance dans le traitement et la prévention des AVC (Ramya et al., 2007 ; Kee et al., 2008).

3.4 Dosage des composés phénoliques totaux et des flavonoïdes : La quantité de phénols totaux des extraits bruts a été déterminée par la méthode colorimétrique de Folin-Ciocalteu selon Singleton et al. (1999) et Heilerová et al. (2003). Le mélange réactionnel a été préparé en mélangeant $1 \mathrm{~mL}$ de solution d'extrait de $0,5 \mathrm{~mL}$ de réactif de Folin-Ciocalteu $(0,5 \mathrm{~N})$ et $1,5 \mathrm{~mL}$ de carbonate de sodium (NaHC03) à 17\%. L'ensemble a été incubé à $37^{\circ} \mathrm{C}$ pendant $30 \mathrm{~min}$ et l'absorbance a été mesurée à $760 \mathrm{~nm}$ contre un blanc sans extrait pris comme référence. Les composés phénoliques totaux ont été quantifiés à partir d'une droite d'étalon de l'acide gallique (0-1000 $\mu \mathrm{g} / \mathrm{mL}$ ) et exprimé en $\mu \mathrm{g}$ équivalent d'acide gallique (EAG/g MS). Le dosage des flavonoïdes totaux a été fait selon la méthode modifiée de Hariri et al., (1991). A $2 \mathrm{~mL}$ de chaque extrait, sont ajoutés $100 \mu \mathrm{L}$ de réactif de Neu. L'absorbance est déterminée à $404 \mathrm{~nm}$ et la teneur en flavonoïdes exprimée en pourcentage $(\%)$.

3.5 Dosage de l'activité antioxydante : Le potentiel antioxydant des extraits a été réalisé in vitro par le test au DPPH (1,1-diphényl-2-picrylhydrazyle). Le protocole est adapté de Blois (1958). 1 mL d'extrait éthanolique, à différentes gammes de concentration et $1,5 \mathrm{~mL}$ d'une

\section{RESULTATS}

4.1 Enquêtes ethnopharmacologiques des préparations médicamenteuses utilisées contre les séquelles des AVC: L'étude des connaissances liées aux AVC, des facteurs de risques et des séquelles a été réalisée auprès de 20 phytothérapeutes. Dix-neuf (95\%) parmi eux reconnaissent et diagnostiquent ces pathologies. Par contre, trois $(15 \%)$ distinguent parfaitement l'AVC hémorragique de l'AVC ischémique. Les solution éthanolique de DPPH $(0,03 \mathrm{mg} / \mathrm{mL})$ ont été soigneusement mélangés et conservés à l'obscurité pendant $30 \mathrm{~min}$. Le blanc a été préparé en remplaçant l'extrait par l'éthanol (1 $\mathrm{mL})$. L'absorbance du mélange réactionnel a été mesurée à $517 \mathrm{~nm}$. Le pourcentage d'inhibition du DPPH a été calculé suivant la formule :

$$
\text { PI } \%=\left[1-\left(\mathrm{A}_{e} / \mathrm{A}_{b}\right)\right] \times 100
$$

$A_{e}:$ absorbance de l'échantillon ;

$A_{b}:$ absorbance du blanc.

Les valeurs de $\mathrm{CI}_{50}$ ont été estimées à partir d'une courbe de pourcentage d'inhibition par rapport à la concentration.

\subsection{Toxicité aigüe des préparations} médicamenteuses : Les essais de toxicité aigüe ont été menés suivant la méthode de " l'ajustement des doses » de la ligne 425 de l'OCDE (OCDE, 2008) et a consisté à tester les extraits bruts en solution aqueuse, à 2000 et 5000 $\mathrm{mg} / \mathrm{kg}$ de poids corporel (pc). La dose d'essai limite de $5000 \mathrm{mg} / \mathrm{kg}$ de pc a été utilisée pour déterminer la DL 50 . La dose de $2000 \mathrm{mg} / \mathrm{kg}$ de pc était l'essai préliminaire. L'évaluation de la toxicité a été réalisée par voie orale, sur 21 lots de trois rattes WISTAR, saines, nullipares et non gravides, âgées de 09 semaines dont les poids variaient de 52 à 86 g. Avant les expériences, les animaux ont été acclimatés aux conditions de laboratoire. Les extraits ont été administrés par voie orale, à l'aide d'une sonde à gavage, le lot témoin recevant de l'eau distillée. La dose de $5000 \mathrm{mg} / \mathrm{Kg}$ de pc correspond à $500 \mathrm{mg} / \mathrm{mL}$ des extraits aqueux pour une ratte pesant $100 \mathrm{~g}$. Les animaux ont été observés durant 14 jours. Les signes cliniques et les mortalités observés ont été notés.

différents diagnostics sont réalisés en milieu hospitalier. Les principales séquelles sont l'hémiplégie $(100 \%)$ et l'aphasie ou trouble de l'élocution $(29,41 \%)$. Les autres séquelles connues sont les troubles de la vision $(11 \%)$ et $\mathrm{du}$ comportement (7\%). L'hypertension artérielle est le principal facteur de risque $(72 \%)$. Les autres facteurs de risque sont le stress $(27,8 \%)$, l'alimentation sodée et le mode de vie 
(21,6\%) et l'obésité (5,56\%). La fatigue, le mal de tête, le tabagisme, l'alcool, la graisse, le diabète, le mauvais cholestérol, etc., ont également été mentionnés comme facteur risque.

\subsection{Caractéristiques}

ethnopharmacologiques des préparations médicamenteuses utilisées contre les séquelles des AVC: Au total 36 préparations médicamenteuses ont été collectées, principalement des solutions, des poudres sèches, des pommades, des huiles, des pâtes (Figure 2, Tableau 1). De façon générale, les préparations sont conditionnées dans des emballages fermés: bouteilles, bidons, boîtes, cartons, sachets plastiques (Figure 2), avec date de péremption et indications thérapeutiques sur des imprimés. Pour des besoins de protection de la propriété intellectuelle, de nombreuses informations ne sont certainement pas communiquées. L'essentiel des produits et préparations proviennent des parties et/ou organes de plantes. Les recettes sont faites par associations de plus de 3 plantes en moyenne (Figure 3). Le décocté est la préparation dominante $(89 \%)$. Les poudres fines représentent $11 \%$. Les préparations sont prescrites en boisson (80\%) et massage (15\%). Le lavement, les bains et les bains de vapeur représentent $5 \%$. La posologie varie d'une préparation à l'autre (Tableau 1). L'hémiplégie est la principale séquelle traitée. Tous les praticiens traitent la paralysie des membres, la déformation du visage et de la bouche. L'hypertension est le traitement secondaire $(11 \%$; Figure 4). Le diabète $(8 \%)$ et les inflammations articulaires suivent. D'autres pathologies comme les douleurs nerveuses, le paludisme, la fatigue générale, les courbatures, le cancer, l'obésité, l'hémorroïde, l'excès de cholestérol, etc., sont également citées. Faire de la marche et mâcher du chewing-gum sont des pratiques conseillées aux patients pour améliorer la consistance des muscles du corps et du visage.

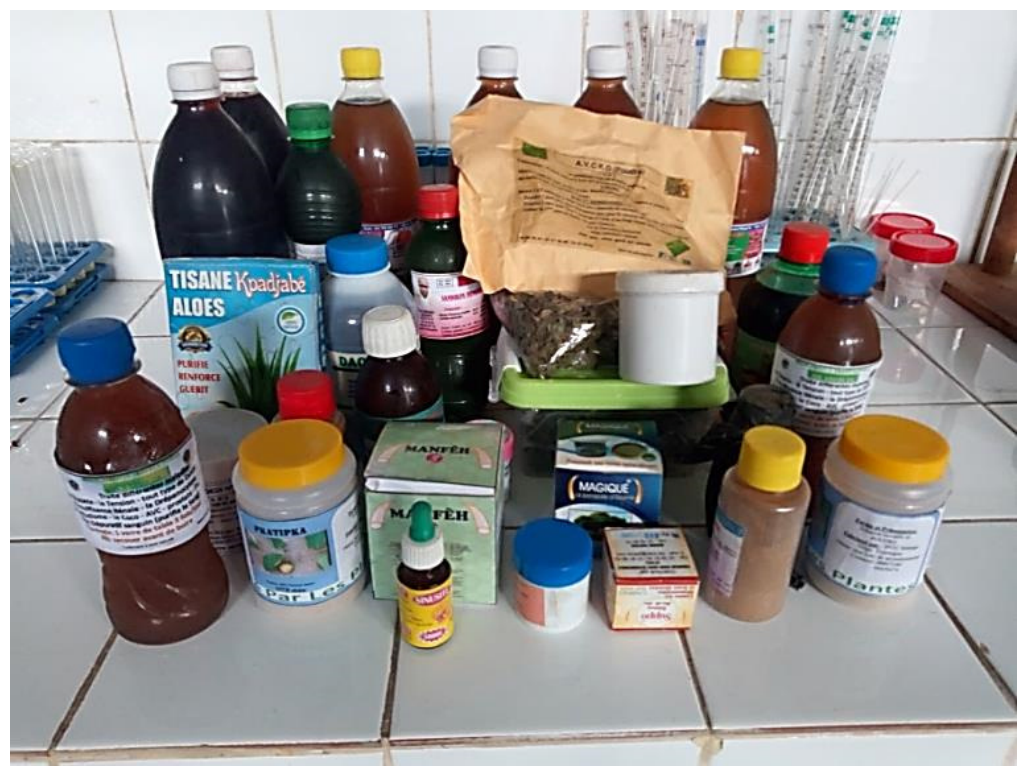

Figure 2: Présentation de quelques préparations médicamenteuses recensées chez les phytothérapeutes 
Tableau 1 : Préparations médicamenteuses recensées chez les phytothérapeutes et indications

\begin{tabular}{|c|c|c|c|c|c|c|}
\hline $\mathbf{N}^{\circ}$ & Préparations & Séquelles & Présentation & $\begin{array}{c}\text { Mode } \\
\text { d'administ. }\end{array}$ & Posologie & Type de trait. \\
\hline $\mathrm{P} 1$ & $\begin{array}{l}\text { Daoutra fort } \\
\text { Daoutra } 3 \\
\end{array}$ & Hémiplégie & $\overline{\text { Décocté }}$ & Voie orale & $\begin{array}{c}1 \text { verre le soir } \\
1 / 2 \text { verre } 2 \mathrm{f} / \mathrm{J}+\text { de la marche }\end{array}$ & $\begin{array}{l}\mathrm{E} \\
\mathrm{E}\end{array}$ \\
\hline $\mathrm{P} 2$ & $\begin{array}{l}\text { Gogniniga Wèrè } \\
\text { Wagnonmon }\end{array}$ & Hémiplégie & Décocté & $\begin{array}{l}\text { Voie orale } \\
\text { Massage } \\
\end{array}$ & $2 \mathrm{f} / \mathrm{J}$ & $\begin{array}{l}\text { E } \\
S\end{array}$ \\
\hline P3 & $\begin{array}{c}\text { Gnamien Djua/Bois } \\
\text { de Dieu }\end{array}$ & Hémiplégie & Décocté & Voie orale & $\begin{array}{l}\text { 1Cuillère } 3 \mathrm{f} / \mathrm{J} \\
\text { pendant } 1 \text { mois }\end{array}$ & $\mathrm{E}$ \\
\hline $\mathrm{P} 4$ & Agengo & Hémiplégie & Décocté & Voie orale & $3 \mathrm{f} / \mathrm{J}$ & $\mathrm{S}$ \\
\hline P5 & Zoe eau de vie & Hémiplégie & Décocté & Voie orale & 1 verre de table $3 \mathrm{f} / \mathrm{J}$ & $E$ \\
\hline P6 & Ballo & Hémiplégie & Macéré & Voie orale & 1 verre de table $2 \mathrm{f} / \mathrm{J}$ & $\mathrm{E}$ \\
\hline $\mathrm{P} 7$ & Kassanguetens & Hémiplégie & Décocté & Voie orale & 2 verres de thé Matin soir & $\mathrm{E}$ \\
\hline P8 & Daloa & Hémiplégie & Décocté & Voie orale & & $\mathrm{E}$ \\
\hline P9 & Mirat de merveille & $\begin{array}{l}\text { Hémiplégie, Fluidifie le sang, } \\
\text { régénérateur }\end{array}$ & Décocté & Voie orale & Particulier à l'individu & $\mathrm{E}$ \\
\hline P10 & $\overline{\mathrm{Nd}}$ & Hémiplégie, Aphasie & Décocté & Voie orale & $\begin{array}{l}1 \text { verre de table } 2 \mathrm{f} / \mathrm{J}+\text { Mâcher du } \\
\text { chewing-gum }\end{array}$ & $\bar{E}$ \\
\hline $\mathrm{P} 11$ & Tisane & Hémiplégie & Infusé & Voie orale & $\begin{array}{c}1 \text { verre de thé } 3 \mathrm{f} / \mathrm{J}+\text { Massage à } \\
\text { base de gingembre }\end{array}$ & $\mathrm{S}$ \\
\hline $\mathrm{P} 12$ & Plantes de la genèse & Hémiplégie & Décocté & Voie orale & $3 \mathrm{f} / \mathrm{J}$ avant le repas & $\mathrm{E}$ \\
\hline$\overline{\mathrm{P} 13}$ & $\begin{array}{c}\text { Monospal } \\
\text { Bio cucurma } \\
\text { Moringa } \\
\text { Elexir } \\
\text { Le vieux champion } \\
\text { Sirop super Bistolet }\end{array}$ & $\begin{array}{l}\text { Hémiplégie } \\
\text { Hémiplégie } \\
\text { Améliore l'immunité } \\
\text { Appétit sexuel } \\
\text { Courbatures } \\
\text { Trouble érectile }\end{array}$ & & $\begin{array}{l}\text { Voie orale } \\
\text { Voie orale } \\
\text { Voie orale } \\
\text { Voie orale } \\
\text { Voie orale } \\
\text { Voie orale }\end{array}$ & $\begin{array}{c}1 \text { verre de thé } 2 \text { à } 3 \mathrm{f} / \mathrm{J} \\
1 \text { cuillère à café } 2 \text { à } 4 \mathrm{f} / \mathrm{J} \\
\text { Voie orale } \\
\text { Voie orale } \\
1 \text { verre de thé } 2 \mathrm{f} / \mathrm{J} \\
\text { Voie orale }\end{array}$ & S \\
\hline$\overline{\mathrm{P} 14}$ & $\begin{array}{c}\text { Préparation spéciale } \\
\text { Heros }\end{array}$ & $\begin{array}{l}\text { Trouble des nerfs } \\
\text { Anémie sévère }\end{array}$ & & $\begin{array}{l}\text { Voie orale } \\
\text { Voie orale } \\
\end{array}$ & $\begin{array}{l}\text { Voie orale } \\
\text { Voie orale } \\
\end{array}$ & $S$ \\
\hline P15 & $\begin{array}{c}\text { Dedi } \mathrm{n}^{\circ} 2 \\
\text { AVC KO } \\
\text { Cannigban } \\
\text { (suppositoire) } \\
\end{array}$ & $\begin{array}{l}\text { Hémiplégie } \\
\text { Hémiplégie } \\
\text { Hémiplégie }\end{array}$ & Décocté & $\begin{array}{c}\text { Voie cutanée } \\
\text { Voie cutanée } \\
\text { Voie anale }\end{array}$ & $\begin{array}{c}\text { Massage } 3 \text { à } 4 \text { min } 3 \mathrm{f} / \mathrm{J} \\
\text { Bain et fumigation } \\
1 \text { boule } 4 \mathrm{f} / \text { semaine dans le rectum }\end{array}$ & $S$ \\
\hline P16 & Thé santé & Hémiplégie & Décocté & Voie orale & 1 verre de table $3 \mathrm{f} / \mathrm{J}$ & $S$ \\
\hline
\end{tabular}




\begin{tabular}{|c|c|c|c|c|c|c|}
\hline P17 & \multicolumn{2}{|c|}{ Epurateur de sang } & & & 3 mois & $\mathrm{S}$ \\
\hline P18 & Kpatikpa & Hémiplégie & Macéré & Voie orale & $1 / \mathrm{J}$ & $\mathrm{S}$ \\
\hline P19 & Kassirime & Hémiplégie, Aphasie & Macéré & & $\begin{array}{l}1 / 2 \text { verre de the dans un verre d'eau } \\
1 \mathrm{f} / \mathrm{J}\end{array}$ & $\bar{E}$ \\
\hline P20 & $\begin{array}{l}\text { Danflah } \\
\text { Manfêh } \\
\text { UCT } \\
\text { Magique } \\
\text { Suppo } \\
\text { Manfêh 1 } \\
\text { N'Lafissou }\end{array}$ & $\begin{array}{l}\text { Troubles des nerfs } \\
\text { Troubles des nerfs } \\
\text { Hypertension } \\
\text { Hémiplégie } \\
\text { Troubles des nerf } \\
\text { Troubles des nerfs } \\
\text { Hémiplégie }\end{array}$ & $\begin{array}{l}\text { Macéré } \\
\text { Décocté } \\
\text { Macéré } \\
\text { Macéré } \\
\text { Décocté } \\
\text { Macératé }\end{array}$ & $\begin{array}{c}\text { Voie cutanée } \\
\text { Voie orale } \\
\text { Voie orale } \\
\text { Voie cutanée } \\
\text { Voie anale } \\
\text { Voie orale } \\
\text { Voie cutanée }\end{array}$ & $\begin{array}{c}\mathrm{f} / \mathrm{J} \text { en massage } \\
1 \text { verre de thé } 2 \mathrm{f} / \mathrm{J} \\
1 \text { verre de table } 3 / \mathrm{J} \\
2 \mathrm{f} / \mathrm{J} \text { en massage } \\
1 \text { boule en suppositoire le soir } \\
1 \text { verre de thé } 2 \mathrm{f} / \mathrm{J} \\
2 \mathrm{f} / \mathrm{J} \text { en massage }\end{array}$ & $\mathrm{S}$ \\
\hline
\end{tabular}

Administ : administration ; S : symptomatique ; E : Etiologique ; J : Jour ; Nd : Non définit 


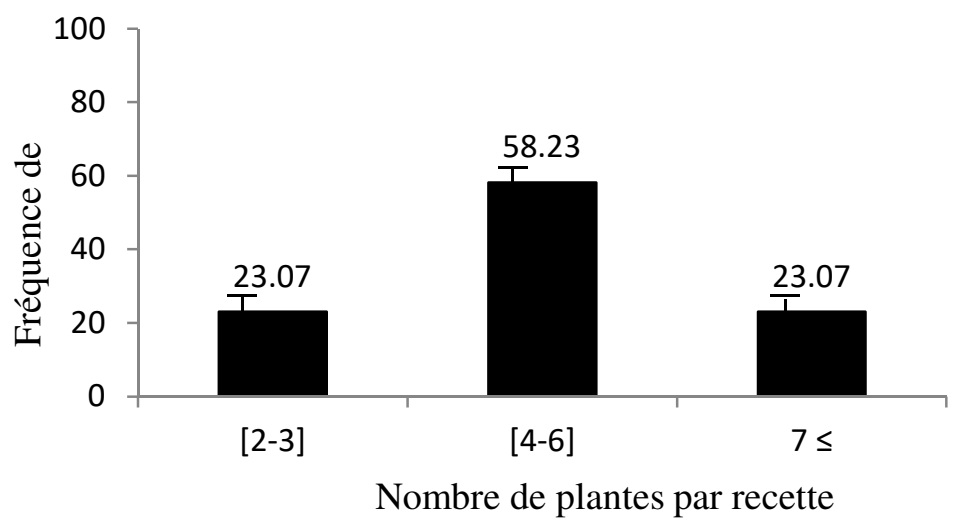

Figure 3 : Histogramme des associations des plantes des préparations médicamenteuses contre les séquelles des AVC recensées au cours de l'étude

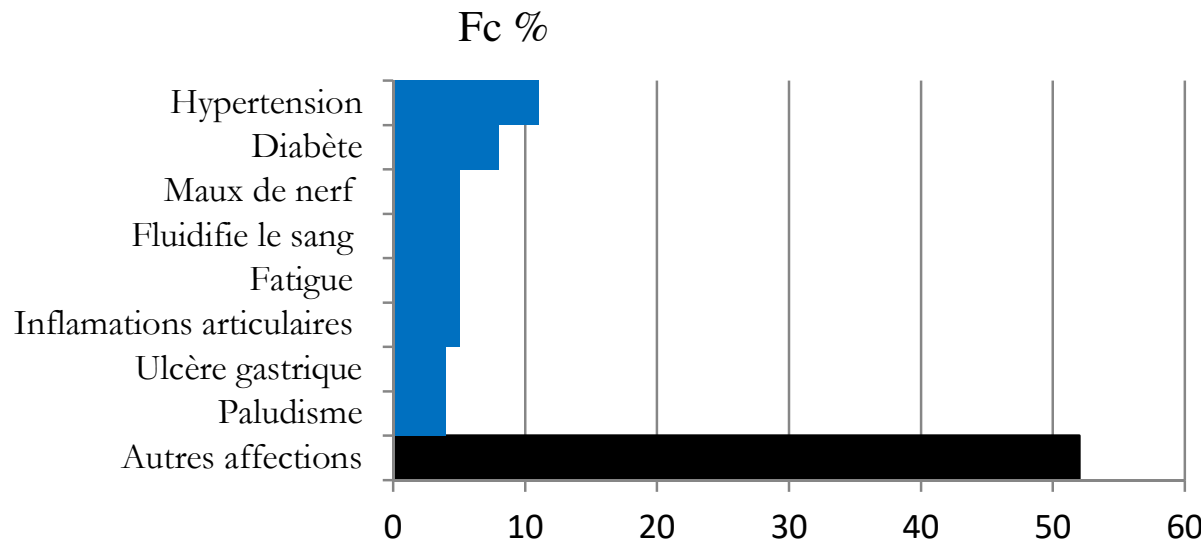

Figure 4 : Autres indications thérapeutiques des préparations médicamenteuses contre les séquelles des AVC
4.3 Criblage phytochimique des préparations médicamenteuses utilisées contre les séquelles des AVC: Dix (10) préparations ont été retenues pour les tests. Il s'agit de Daoutra fort, Daoutra 3, Gogniniga wèrè, Gnamien Djua, Agengo, Zoe eau de vie, Ballo, Kassanguetens, Daloa et Mirat de merveille (Tableau 1). Les résultats du criblage
indiquent qu'elles contiennent toutes des polyphénols et des flavonoïdes (Tableau 2). Les tanins, les saponosides et les stérols sont présents dans 7 préparations puis les alcaloïdes dans 6. La préparation la plus riche en composés phytochimiques Daloa, 7 sur les 7 recherchés. Les plus pauvres sont Agengo et Mirat de merveille. phytochimique réalisé sur ces préparations 
Tableau 1 : Composition des préparations médicamenteuses des phytothérapeutes utilisées contre les séquelles des AVC

\begin{tabular}{l|l|l|l|l|l|l}
\hline \multirow{2}{*}{$\begin{array}{l}\text { Préparations } \\
\text { médicamenteuses }\end{array}$} & \multicolumn{9}{|c}{ Composition } \\
\cline { 2 - 7 } & Polyphénols & Flavonoïdes & Stérols & Saponosides & Tanins & Alcaloïdes \\
\hline Daloa & + & +++ & ++ & +++ & ++ & ++ \\
Ballo & +++ & ++ & ++ & - & ++ & +++ \\
Daoutra 3 & +++ & + & - & ++ & ++ & + \\
Gogniniga wèrè & + & + & ++ & +++ & ++ & +++ \\
Kassanguetens & +++ & + & ++ & +++ & ++ & + \\
Gnamien Djua, & + & ++ & + & + & ++ & - \\
Eau de Vie & +++ & ++ & ++ & ++ & - & - \\
Daoutra F & ++ & ++ & - & - & +++ & - \\
Mirat de merveille & + & + & - & + & - & - \\
Agengo & + & + & + & - & - & ++ \\
\hline
\end{tabular}

Signification des symboles : + : Présent $;++$ : Abondamment présent ; $+++:$ Très abondamment présent ; - : Absence

\subsection{Dosages des polyphénols et des flavonoïdes totaux préparations médicamenteuses utilisées contre les} séquelles des AVC : L'évaluation des teneurs en polyphénols des préparations (Tableau 3) indique qu'elles varient significativement d'une préparation à l'autre $(\alpha=0,05 ; P<0,0001)$, entre 1765 et $186356 \mu \mathrm{g}$ EAG/g MS. Daoutra $\mathrm{F}(173913,8 \mu \mathrm{g}$ EAG/g MS) et Daoutra 3 (151456,9 $\mu \mathrm{g}$ EAG/g MS) ont présenté les teneurs les plus élevées. Ballo, Daloa et eau de vie Zoé ont des teneurs moyennes, comprises entre 84000 et $40000 \mu \mathrm{g}$ EAG/g MS. Mirat de merveille, Agengo, et Kassanguetens ont présenté des teneurs comprises entre 16000 et $28000 \mu \mathrm{g}$ EAG/g MS. Gnamien Djua et Gogniniga Wèrè ont présenté de très faibles teneurs en polyphénols, 6 733,33 et $1764,94 \mu \mathrm{g}$ $\mathrm{EAG} / \mathrm{g} \mathrm{MS}$, respectivement. L'étude des teneurs en flavonoïdes totaux des préparations a montré qu'elles varient très significativement $(\alpha$ $=0,05 ; P<0.0001)$ également (Tableau 4). Selon les résultats obtenus, Daoutra Fort et Daoutra 3 ont présenté les teneurs les plus élevées, $77,13 \pm 0,71$ et 75,07 $\pm 0,65 \%$, respectivement. Les teneurs des flavonoïdes les plus faibles ont été observées dans Gnamien Djua (5,39\%) et Mirat de merveille (4,22\%).

Tableau 3 : Teneurs en polyphénols totaux des préparations médicamenteuses utilisées contre les séquelles des AVC

\begin{tabular}{|c|c|c|}
\hline Préparations médicamenteuses & Teneurs $(\mu \mathrm{g}$ EAG $/ g$ MS) $\pm \mathrm{SD}$ & Paramètres statistiques \\
\hline $\begin{array}{l}\text { Daoutra Fort } \\
\text { Daoutra } 3 \\
\text { Ballo } \\
\text { Daloa } \\
\text { Eau de vie Zoé } \\
\text { Mirat de merveille } \\
\text { Agengo } \\
\text { Kassanguetens } \\
\text { Gnamien Djua } \\
\text { Gogniniga Wèrè }\end{array}$ & $\begin{array}{l}186356,32 \pm 2157^{\mathrm{a}} \\
149545,98 \pm 3712^{\mathrm{b}} \\
83080,46 \pm 29146^{\mathrm{c}} \\
51672,41 \pm 6355^{\mathrm{d}} \\
40005,75 \pm 263^{\mathrm{de}} \\
27850,57 \pm 1210^{\mathrm{fg}} \\
20695,40 \pm 2056^{\mathrm{g}} \\
16385,06 \pm 1811^{\mathrm{g}} \\
6733,33 \pm 680,5^{\mathrm{h}} \\
1764,94 \pm 54,74^{\mathrm{e}}\end{array}$ & $\begin{array}{ll}\mathrm{dl} & 10 \\
F & 85,97 \\
P & 0,0001\end{array}$ \\
\hline
\end{tabular}


Tableau 4: Teneur en flavonoïdes totaux des préparations médicamenteuses utilisées contre les séquelles des AVC

\begin{tabular}{|c|c|c|}
\hline Préparations médicamenteuses & \% Flavonoïdes & Paramètres statistiques \\
\hline $\begin{array}{l}\text { Daoutra Fort } \\
\text { Daoutra } 3 \\
\text { Eau de VieZoé } \\
\text { Daloa } \\
\text { Ballo } \\
\text { Kassanguetens } \\
\text { Agengo } \\
\text { Gogniniga Wèrè } \\
\text { Gnamien Djua } \\
\text { Mirat de merveille }\end{array}$ & $\begin{array}{l}77,13 \pm 0,71^{\mathrm{a}} \\
75,07 \pm 0,65^{\mathrm{a}} \\
23,41 \pm 0,89^{\mathrm{b}} \\
20,92 \pm 1,49^{\mathrm{bc}} \\
16,73 \pm 1,29^{\mathrm{c}} \\
12,19 \pm 1,69^{\mathrm{e}} \\
11,62 \pm 1,18^{\mathrm{e}} \\
6,24 \pm 0,42^{\mathrm{f}} \\
4,11 \pm 0,18^{\mathrm{g}} \\
0,567 \pm 0,08^{\mathrm{h}}\end{array}$ & $\begin{array}{ll}\mathrm{dl} & 10 \\
F & 1508 \\
P & 0,0001\end{array}$ \\
\hline
\end{tabular}

4.5 Evaluation du potentiel antioxydant des préparations médicamenteuses utilisées contre les séquelles des AVC : L'évaluation du potentiel antioxydant des différentes préparations médicamenteuses a montré qu'il varie significativement $(\alpha=0,05 ; P<0.0001)$ d'une préparation à l'autre (Tableau 5). L'étude de l'activité anti-radicalaire a indiqué plusieurs catégories de préparations. Un potentiel antioxydant important a été obtenu avec Ballo, Daoutra Fort et Daoutra 3. Les $\mathrm{CI}_{50}$ de 0,0280 ; 0,0409 et $0,0593 \mathrm{mg} / \mathrm{mL}$ observées indiquent que ces préparations présentent les meilleures activités antioxydantes. Cette activité est moyenne avec les préparations Eau de Vie Zoé, Agengo et Gnamien Djua.
4.6 Toxicité aigüe des préparations médicamenteuses utilisées contre les séquelles des AVC Les tests de toxicité aiguë réalisés à partir des préparations médicamenteuses n'ont présenté, pour l'essentiel, aucun signe de toxicité aiguë, mis à part quelques signes de toilettage, d'apathie, d'excitation et de toux avec Daoutra 3 (Tableau 6). Pour les 10 préparations testées à 2000 $\mathrm{mg} / \mathrm{kg}$ et $5000 \mathrm{mg} / \mathrm{kg}$ de PC, tous les animaux gavés ont survécu après les 14 jours d'observation. Les résultats indiquent des $\mathrm{DL}_{50}$ supérieures à $5000 \mathrm{mg} / \mathrm{kg}$ de pc. Selon les résultats obtenus, toutes ces préparations peuvent être considérées non toxiques.

Tableau 5 : $\mathrm{CI}_{50}$ des préparations médicamenteuses utilisées contre les séquelles des AVC

\begin{tabular}{|c|c|c|}
\hline Préparations médicamenteuses et témoin & $\mathrm{CI}_{50}(\mathrm{mg} / \mathrm{mL}) \pm \mathrm{SD}$ & Paramètres statistiques \\
\hline Vitamine $\mathrm{C}$ & $0,0054 \pm 0,0002^{\mathrm{a}}$ & \\
\hline Ballo & $0,0280 \pm 0,0008^{\mathrm{b}}$ & \\
\hline Daoutra Fort & $0,0409 \pm 0,0001^{\mathrm{c}}$ & \\
\hline Daoutra 3 & $0,0593 \pm 0,0035^{\mathrm{c}}$ & 10 \\
\hline Eau de Vie Zoé & $0,2528 \pm 0,0006^{\mathrm{ef}}$ & 10210 \\
\hline Agengo & $0,3066 \pm 0,0049^{\mathrm{f}}$ & 0,0001 \\
\hline Gnamien Djua & $0,3787 \pm 0,0014^{\mathrm{g}}$ & \\
\hline Daloa & $2,331 \pm 0,0012^{\mathrm{h}}$ & \\
\hline Mirat de merveille & $2,767 \pm 0,0176^{\mathrm{h}}$ & \\
\hline Kassanguetens & $14,547 \pm 0,2464^{\mathrm{i}}$ & \\
\hline Gogniniga Wèrè & $21,231 \pm 0,3567^{i}$ & \\
\hline
\end{tabular}


Tableau 6 : Toxicité aigüe des préparations médicamenteuses utilisées contre les séquelles des AVC

\begin{tabular}{l|l|l|l|l|l|l}
\hline \multirow{2}{*}{$\begin{array}{l}\text { Préparations médicamenteuses } \\
\text { et témoin }\end{array}$} & \multicolumn{7}{|c}{ Signes cliniques } \\
\cline { 2 - 7 } Eau distillée & Toilettage & Apathie & Excitation & Toux & Mortalité & Autres \\
Ballo & - & - & - & - & - & - \\
Mirat de merveille & ++ & - & - & - & - & - \\
Daloa & ++ & - & - & - & - & - \\
Kassanguetens & ++ & + & - & - & - & - \\
Agengo & ++ & + & - & - & - & - \\
Daoutra Fort & + & - & + & + & - & - \\
Eau de Vie & + & - & + & + & - & - \\
Gnamien Djua & + & + & + & - & - & - \\
Gogniniga Wèrè & + & + & + & - & - & - \\
Daoutra 3 & + & + & + & - & - & - \\
\hline & + & + & + & + & - & - \\
\hline
\end{tabular}

+ : présence; - : absence; Autres : Apathie, refus de nourriture, saignement buccal, saignement nasal, coma, diarrhée, tremblement, convulsion, mortalité

\section{DISCUSSION}

L'évaluation des connaissances relatives aux $\mathrm{AVC}$, à leurs facteurs de risque et séquelles, montre que très peu de phytothérapeutes distinguent un AVC hémorragique d'un AVC Ischémique. Le caractère plus ou moins ressent de l'émergence de cette maladie (Feigin et al., 2014) en Côte d'Ivoire et le faible niveau d'instruction des phytothérapeutes pourraient justifier ces résultats. Pour les phytothérapeutes, l'hypertension artérielle représente la principale cause des AVC. Des études cliniques récentes en Côte d'Ivoire (N'Goran et al., 2015 ; Diarra et al., 2016) et en Afrique (Coulibaly et al., 2010) confirment ce résultat. D'autres facteurs, tous aussi importants, comme l'alcoolisme et le diabète sont également cités (N'Goran et al., 2015 ; Diarra et al., 2016). Les principales séquelles signalées par les phytothérapeutes sont celles notées par la médecine moderne (Hendricks et al., 2002), indiquant, ainsi, le niveau d'appréciation de ces Praticiens de Médecine Traditionnelle qui ont choisi cette spécialité assez complexe de la santé humaine. Les préparations médicamenteuses proposées sont, pour la plupart, des solutions. Les patients actuels sont plutôt attirés par ce type de préparations médicamenteuses, d'où le développement de nombreux cabinets de médecine traditionnelle. Elles sont davantage utilisées dans le traitement de l'hémiplégie qui représente la séquelle la plus apparente et invalidante des AVC (Murray et Lopez, 1997 ; Hendricks et al., 2002). Elles le sont, également, pour de nombreuses autres pathologies (hypertension artérielle, diabète, paludisme, etc.), qui rendent précaires la santé et le mode de vie du patient. Selon HAS (2014), les médicaments utilisés dans la prévention secondaire permettent de réduire le risque d'un nouvel événement vasculaire (cérébral ou myocardique) et aident à la guérison du patient. De l'analyse phytochimique, il ressort la présence relativement fréquente de nombreux composés phytochimiques. Parmi les plus présents, les polyphénols, les flavonoïdes et les tanins sont importants dans le traitement et la prévention des maladies cardiovasculaires, ce qui permet de faire des prédictions quant à leurs intérêts thérapeutiques dans le traitement des séquelles des AVC. Les polyphénols ont des propriétés antioxydantes, vasodilatatrices, antithrombotiques et anti-inflammatoires (Manach et al., 2005). Les flavonoïdes sont cardioprotecteurs (Hooper et al., 2008 ; Lotito et Frei, 2006), antihypertensifs (Chen et al., 2009) et certaines flavones sont antiplaquettaires (O'Leary et al., 2004). De nombreux tanins sont antithrombotiques et/ou anticoagulants (Kee et 
al., 2008). Tous ces métabolites présentant des activités antioxydantes et sont indispensables pour résorber la lésion cérébrale grâce à leur capacité à piéger les radicaux libres et à réduire le stress oxydant produit après un AVC, par régénération des cellules du cerveau (Quintard, 2014). Plusieurs études chez des modèles animaux et chez des sujets humains ont effectivement confirmé la biodisponibilité des phénols à exercer un rôle protecteur contre le stress oxydatif et les dommages des radicaux libres (Prior et al., 2003 ; Szajdek et al., 2008). L'étude de la teneur en polyphénols et flavonoïdes, et de l'activité antioxydante a permis de comparer et de classer les préparations médicamenteuses selon leur potentiel à traiter les séquelles des AVC. Certaines sont très riches en polyphénols (Daoutra Fort et Daoutra 3), en Flavonoïdes (Daoutra Fort et Daoutra 3) et

\section{CONCLUSION}

La présente étude menée auprès des phytothérapeutes affiliés au Programme National de Promotion de la Médecine Traditionnelle en Côte d'Ivoire, montrent que ces praticiens ont des connaissances relativement bonnes sur les AVC, leurs facteurs de risques et leurs séquelles, puis proposent de nombreux préparations médicamenteuses contre ces dernières. Le facteur de risque des AVC le plus cité est l'hypertension artérielle et la séquelle la plus observée est l'hémiplégie. 36 préparations médicamenteuses ont été recensées au cours de la présente étude. La caractérisation phytochimique de dix de ces préparations a noté

\section{REMERCIEMENTS}

Les auteurs de ce manuscrit remercient l'ASCAD (Académie des Sciences, des Arts, des Cultures d'Afrique et des Diasporas Africaines) pour sa contribution financière à cette étude, le

\section{REFERENCES BIBLIOGRAPHIQUES}

Beghdad MC, Benammar C, Bensalah F, Sabri FZ, Belarbi M. et Chemat F: 2014. Antioxidant activity, phenolic and flavonoid content in leaves, flowers, présente un potentiel antioxydant remarquable (Korhogo, Daoutra Fort et Daoutra 3). Les teneurs en polyphénols sont bien supérieurs à celles observées dans de nombreuses études pour de nombreuses plantes à fort potentiel (Djeridane et al., 2006 ; Ksouri, 2007 ; Beghdad et al., 2014). Ces résultats sont indicateurs de la qualité des préparations médicamenteuses des phytothérapeutes ainsi que de la vitalité de la médecine traditionnelle en Côte d'Ivoire. L'étude de la toxicité aiguë des différentes préparations médicamenteuses par voie orale chez les rats Wistar n'a présenté aucun mort au terme des 14 jours d'observation. Les 10 préparations proposées aux patients contre les séquelles des AVC peuvent déjà, à ce stade des investigations, être considérés comme potentiellement non toxiques pour la santé humaine.

clairement leur richesse en polyphénols, flavonoïdes, tanins, saponosides, stérols et alcaloïdes, métabolites secondaires impliqués dans le traitement et la prévention des maladies cardiovasculaires. Les tests de toxicité qui ont suivi ont montré qu'elles sont potentiellement non toxiques pour la santé des patients. L'étude a, en outre montré que certaines préparations (Daoutra fort, Daoutra, Ballo) particulièrement riches en polyphénols, en flavonoïdes et présentant une activité antioxydante appréciable présentent les meilleurs profils dans le traitement des séquelles des AVC.

Programme National de Promotion de la Médecine Traditionnelle en Côte d'Ivoire et tous les phytothérapeutes. 
(3): $\quad 486-491 . \quad \underline{\text { DOI: }}$ $10.5897 / \mathrm{AJB} 2013.12833$

Blois M: 1958. Déterminations antioxydantes par l'utilisation d'un radical libre stable. Nature 181, 1199-

1200. https://doi.org/10.1038/1811199 $\underline{\mathrm{a} 0}$

Bouleti C, Mathivet T, Lapergue B, Monnot C. et Germain S : 2014. Accident vasculaire cérébral Protection de l'intégrité vasculaire lors de la réperfusion. NOUVELLE $m / s n^{\circ} 6-7$, vol. 30. DOI : $10.1051 / \mathrm{medsci} / 20143006003$

Chen J, Du CY, Lam KY, Zhang WL, Lam CT, Yan AL, Yao P, Lau DT, Dong TT. et Tsim KW: 2014. The standardized extract of Ziziphus Jujuba fruit (jujube) regulates pro-inflammatory cytokine expression in cultured murine macrophages: suppression of lipopolysaccharide-stimulated NF-kB activity. Phytother Res., 28 (10): 1527-32. https://doi.org/10.1002/ptr.5160.

Coulibaly S, Diakité S, Diall IB, Menta I, Sacko AK et Diallo B: 2010. Cerebrovascular accidents: risk factors, clinical course and prognosis in the Cardiology "B" Service of the du Point $G$ University Hospital Center, Bamako]. Mali Med., 25(1):32-6. DOI: 10.11604 / pamj.2015.21.160.6852

Daud N, Hashim H. et Samsulriza N: 2013. Anticoagulant Activity of Averrboa bilimbi Linn in Normal and Alloxan-Induced Diabetic Rats The Open Conference Proceedings Journal, 4, (Suppl-2, M6) 21-26. DOI: 10.2174 / 2210289201304020021

Diarra EAA, Assouan KAE, Yao BR, Kouame KL, Kajo C. et Tanoh C: 2016 : Épidémiologie des AVC en Côte d'Ivoire et perspectives. Revue Neurologique, 172(1), A164. https://doi.org/10.1016/j.neurol.2016. 01.391

Dickneite G, Seiffe D, Diehl KH, Rogers M. et Czech J: 1995. Pharmacological characterization on a new 4amidinophenyl-alanine thrombin- inhibitor (CRC220). Thromb. Res. 77: 357368.:https://doi.org/10.1016/00493848(95)93839-R

Djeridane A, Yousfi M, Nadjemi B, Vidal N, Lesgards JF. et Stocker P: 2007. Screening of some A Algerian medicinal plants for the phenolic compounds and their antioxidant activity. European Fooda Research and Technology. 224: 801-809. http://dx.doi.org/10.1007/s00217-006$\underline{0361-6}$

Feigin VL, Forouzanfar MH, Krishnamurthi R, Mensah GA, Connor M, Bennett DA, Moran AE, Sacco RL, Anderson L, Truelsen T, O’Donnell M, Venketsubramanian N, Barker-Collo S, Lawes CMM, Wang W, Shinohara Y, Witt E, Ezzati M, Naghavi M. et Murray C: 2014. Global and regional burden of stroke during 1990-2010: findings from the Global Burden of Disease Study 2010. Lancet, 383(9913): 245-255. DOI: 10.1016/s0140-6736(13)61953-4

Go AS, Mozaffarian D. et Roger VL: 2014. Heart disease and stroke statistics-update: a report from the american heart association. Circulation, 129(3) : 28-292. https://doi.org/10.1161/01.cir.0000441 $\underline{139.02102 .80}$

Harbome JB: 1998. Phytochemical methods. In: A Guide to Modern Techniques of Plant Analysis. 3rd ed. New York, NY: Chapman and Hall; 1998. p. 40-137.

Hariri EB, Sallé G. et Andary C: 1991. Involvement of flavonoids in the resistance of two popular cultivars to mistletoe (Viscum album L.), Protoplasma, 162(1) : 20-26. DOI: $10.1007 /$ $\underline{\mathrm{BF} 01403897}$

HAS : 2014. Prévention vasculaire après un infarctus cérébral ou un accident ischémique transitoire. Méthode recommandation pour la pratique clinique. Haute Autorité de Santé, 221 p. www.has-sante.fr

Heilerová L, Bučková M, Tarapčík P, Šilhár S. et Labuda J : 2003. Comparaison des 
données d'activité antioxydante pour les extraits aqueux de mélisse (Melissa officinalis L.), d'origan (Origanum vulgare L.) , le thym ( Thymus vulgaris L.) et l'agrimonie (Agrimonia eupatoria L.) obtenus par des méthodes conventionnelles et le biocapteur à base d'ADN. Czech J. Food Sci., 21: 78-84. https://doi.org/10.17221/3480-CJFS

Hendricks HT, Van Limbeek J, Geurts AC. et Zwarts MJ: 2002. Motor recovery after stroke: A systematic review of the literature. Arch. Phys. Med. Rehabil. 83: 1629-1637.

https://doi.org/10.1053/apmr.2002.35 473

Hooper L, Kroon PA, Rimm EB, Cohn JS, Harvey I, Le Cornu KA, Ryder JJ, Hall WL. et Cassidy A : 2008. Flavonoids, flavonoid-rich foods, and cardiovascular risk: A meta-analysis of randomized controlled trials. Am. J. Clin. Nutr., 88: 38-50.

https://doi.org/10.1093/ajcn/88.1.38

Kawakami K, Aketa S, Sakai H, Watanabe Y, Nishida H. et Hirayama M: 2011. Antihypertensive and vasorelaxant effects of water-soluble proanthocyanidins from Persimmom leaf tea in spontaneously hypertensive rats. Biosci. Biotechnol. Biochem, 75 : 1435-1439. https://doi.org/10.1271/bbb.100926

Kee NLA, Mnonopi N, Hajierah D, Naudé RJ. et Frost CL: 2008. Antithrombotic/anticoagulant and anticancer activities of selected medicinal plants from South Africa. African Journal of Biotechnology Vol. 7 (3) : 217-223. https://doi.org/10.5897/AJB07.834

Ksouri R, Megdiche W, Debez A, Falleh H, Grignon C. et Abdelly C: 2007. Salinity effects on polyphenol content and antioxidantactivities in leaves of the halophyte Cakil emaritima. Plant. Physiol Bioch, 45:

244-249. https://doi.org/10.1016/j.plaphy.2007. $\underline{02.001}$

Leys D. et Cordonnier C : 2009. Traitements des accidents vasculaires cérébraux en phase aigüe et prévention secondaire. Med Sci (Paris), $25: 733-8$.

https://doi.org/10.1051/medsci/20092 $\underline{\text { 58-9733 }}$

Lotito SB. et Frei B: 2006. Consumption of flavonoid-rich foods and increased plasma capacity in humans: Cause, consequence, or epiphenomenon? Free Radic. Biol. Med., 41: 1727-1746. https://doi.org/10.1016/j.freeradbiome d.2006.04.033

Luna-Vázquez FJ, Ibarra-Alvarado C, RojasMolina A, Rojas-Molina JI, Yahia EM, Rivera-Pastrana DM, Rojas-Molina A. et Zavala-Sánchez MA : 2013. Nutraceutical Value of Black Cherry Prunus serotina Ehrh. Fruits: Antioxidant and Antihypertensive Properties. Molecules 2013, 18, 14597-14612. Doi:10.3390/molecules181214597

Manach C, Williamson G, Morand C, Scalbert A. et Remesy C: 2005. Bioavailability and bioefficacy of polyphenols in humans. I. Review of 97 bioavailability studies. $A m$. J. Clin. Nutr., 81: 230S-242S. https://doi.org/10.1093/ajcn/81.1.230 $\mathrm{S}$

Murray CJ. et Lopez AD : 1997. Motality by cause for eight regions in the world: Global Burden of Disease Study, Lancet: 1269-127. https://doi.org/10.1016/S01406736(96)07493-4

N'Goran KNYY, Traoré F, Tano M, Kramoh KE, Kakou AJB, Konin C. et Kakou GM: 2015. Aspects épidémiologiques des Accidents Vasculaires Cérébraux (AVC) aux urgences de l'institut de cardiologie d'Abidjan (ICA). Pan African Medical Journal, 21(160), 5 p. DOI : $10.11604 /$ pamj.2015.21.160.6852

OCDE : 2008. Pharmacopée européenne. 6eme édition, Tome1. Pp. 178-568. 
O’Leary KA, Pascual-Tereasa S, Needs PW, Bao YP, O’Brien NM. et Williamson G: 2004. Effect of flavonoids and vitamin $\mathrm{E}$ on cyclooxygenase- 2

(COX-2) transcription. Muta. Res. Fund. Mol. Mech. Mut., 551: 245-254. DOI: $10.1016 / \mathrm{i} . \mathrm{mrfmmm} .2004 .01 .015$

Prior RL: 2003. Fruits and vegetables in the prevention of cellular oxidative damage. Am. J. Clin. Nutr., 78: 570S-578S. https://doi.org/10.1093/ajcn/78.3.570 $\mathrm{S}$

Proteggente AR, Pannala AS, Paganga G, van Buren L, Wagner E, Wiseman S, Van de Put F, Dacombe C. et Rice-Evans C: 2002. The antioxidant activity of regularly consumed fruit and vegetables reflects their phenolic and vitamin $\mathrm{C}$ composition. Free Radic. Res. 2002, 36, 217-233.

https://doi.org/10.1080/107157602900 06484

Quintard H: 2014. Etude des propriétés neuroprotectrices et neurorégénératives du MLC901, issu de la Médecine Traditionnelle Chinoise face à l'ischémie globale et au traumatisme crânien chez le rongeur. Sciences agricoles. Université Nice Sophia Antipolis, 206 p.

Ramya D, Thirunavukkarasu P, Barathi A. et Asha S: 2017. In vitro anticoagulant activity of Nelumbo nucifera leaf extracts on normal healthy blood plasma. International Journal of Green Pharmacy, 11

(3) : 16.

DOI: http://dx.doi.org/10.22377/ijgp. v11i03.1121

Singleton V, Orthofer R. et Lamuela-raventos RM: 1999. Analysis of total phenols and other oxidation substrates and antioxidants by means of FolinCiocalteu reagent. Methods Enzymol. 299 : 152-178.

https://doi.org/10.1016/S00766879(99)99017-1

Szajdek A. et Borowska EJ: 2008. Bioactive compounds and health-promoting properties of berry fruits: A review. Plant Foods Hum. Nutr., 63, 147-156. DOI 10.1007/s11130-008-0097-5

Tanelli F, Belardo TG, Peiro CHF, Gama RMD, Fonseca FLA. et José Armando-Junior $\mathrm{J}$ : 2017. Preliminary Studies on Anticoagulation and Antiplatelet Activities of Plinia cauliflora (Mart.) Kausel Leaves Extract Journal of Applied Pharmaceutical Science, 7 (04) : 115-118.

DOI: $\underline{10.7324 / J A P S .2017 .70416}$ 